\title{
Pain-related Disruptive Behavior during Dental Treatment Interpreted as Uncooperative Behavior-Small Case Series
}

\author{
Ashkenazi Malka ${ }^{1}$, Gotler Maya ${ }^{2}$
}

\begin{abstract}
Aim:To describe a small series of six cases of children who were diagnosed as uncooperative and referred to general anesthesia to complete their dental treatment. These children were actually exhibiting pain-related disruptive behaviors during previous dental treatments; we determined the reasons for ineffective anesthesia.

Background: One of the most common reasons for disruptive behaviors in children during operative dental treatment is the experiencing of pain during treatment. Disruptive behavior may lead to treatment under general anesthesia.

Case description: Parents of six uncooperative children referred to general anesthesia for dental treatment, arrived at our clinic, because they wanted a second opinion. The children were found to be nonresponsive to the common anesthetic technique and were treated by several approaches to increase the effectiveness of anesthesia. These included supplementary anesthesia to accessory innervation (in three cases), changing brands of anesthetic (in two cases), injecting the maximal dose at once to prevent tachyphylaxis, and waiting 5 minutes to achieve effectiveness of anesthesia (in one case). After achieving effective anesthesia, all the children fully cooperated during the operative treatment. Conclusion: Ineffective anesthesia can lead to severe disruptive behavior when continuing the treatment irrespective to the sensation of pain. Several approaches were used to increase the effectiveness of anesthesia resulting in cooperation of the pediatric patients.

Clinical significance: Behavioral response to ineffective anesthesia may be diagnosed as uncooperativeness. Effort should be put to differentiate between ineffective anesthesia and uncooperative patient.
\end{abstract}

Keywords: Case series study, Ineffective anesthesia, Lidocaine, Local anesthesia.

International Journal of Clinical Pediatric Dentistry (2019): 10.5005/jp-journals-10005-1618

\section{BACKGROUND}

One of the most common reasons for disruptive behaviors in children during operative dental treatment is the experiencing of pain during treatment. ${ }^{1-3}$ Nakai et al. found that $11.6 \%$ of the children experienced pain during dental treatment. ${ }^{4}$ Kaufman et al. reported that $13 \%$ of dentists experienced failure in anesthesia during 5 days' work, and subsequently $10 \%$ of the treatments were discontinued, as a result of insufficient local anesthesia. ${ }^{5}$

Several reasons, related to the dentist, may contribute to existing pain during operative dental treatment. These include: relying on nitrous oxide analgesia for anesthesia, not anesthetizing the palatal/lingual gingiva when placing a rubber dam's clasp, or when extracting teeth and administering an insufficient dose of local anesthesia. Moreover, the local anesthetic dosage limit allowed for injection to young pediatric patients may exacerbate the occurrence of insufficient anesthesia. The inaccurate technique, especially during mandibular block injection, can also induce insufficient anesthesia. Another aspect of inaccurate injection technique is tachyphylaxis, defined as an increasing tolerance to a repeatedly administered drug. The reason for this phenomenon is attributed to the development of edema, reduced spread of the anesthesia, localized hemorrhage, clot formation, hypernatremia, and decreased tissue $\mathrm{pH}^{6}$

Notwithstanding, it should be emphasized that treatment of specific teeth was found associated with frequent failure of anesthesia, irrespective to the technique of injection; these include the lower permanent first molar following inferior alveolar nerve block, ${ }^{5,7-9}$ primary upper second molar, upper first permanent molars, ${ }^{10}$ and upper permanent central incisors. ${ }^{11}$ The unsuccessful anesthesia in these cases is mainly attributed to the anatomical variation in the innervation of these teeth. ${ }^{12}$
1,2Multidisciplinary Dental Clinic, Petah Tikva, Israel

Corresponding Author: Ashkenazi Malka, Multidisciplinary Dental Clinic, Petah Tikva, Israel, e-mail: malka.ashkenazi@gmail.com

How to cite this article: Malka A, Maya G. Pain-related Disruptive Behavior during Dental Treatment Interpreted as Uncooperative Behavior-Small Case Series. Int J Clin Pediatr Dent 2019;12(4):347-351.

Source of support: Nil

Conflict of interest: None

Typical supplementary anesthesia required following ineffective mandibular block injection, infiltration to upper primary or permanent molars or to upper central incisors include long buccal and/or Intraligamental injection, ${ }^{8,9,13-16}$ posterior superior alveolar nerve block or greater palatine nerve block ${ }^{16-19}$ and incisive canal nerve block and/or Intraligamental anesthesia, respectively, preferably administered by a computerized delivery system. . $^{11,20,21}$

Another group of teeth that are usually associated with ineffective anesthesia, regardless to the injection technique, are teeth with pulpitis or with developmental disturbances, such as molar incisor hypo-mineralization (MIH). ${ }^{22,23}$

The aim of this article is to describe six cases of children who were referred to our dental clinic because of severe disruptive behavior during previous dental treatments. Some children were even referred to general anesthesia (GA), but their parents came to ask for a second opinion. The reason for these children's disruptive behavior was found to be an ineffective anesthesia. 


\section{Case Description}

\section{Changing Brand of Anesthetics (Two Case Reports)} Case 1

GD a 5.5-year-old girl arrived at our dental clinic for a second opinion. Her father reported that she was referred to undergo dental treatment under GA because of her inability to cooperate. During clinical examinations, DG was withdrawn and fearful but followed the instructions even during the performance of intraoral radiographs. Accordingly, it was decided to treat her under nitrous oxide analgesia $\left(50 \% \mathrm{~N}_{2} \mathrm{O} / 50 \% \mathrm{O}_{2}\right)$. On the first operative treatment ( 5 weeks later), we planned to treat tooth 74 by pulpotomy and preformed stainless-steel crown, and 75 by proximal [mesioocclusal (MO) restoration] composite restoration. The teeth were anesthetized by $1 \frac{1 / 4}{4}$ cartridge of lidocaine $2 \%$ with $1: 100,000$ epinephrine which was injected by a Computer-controlled local anesthetic delivery system (C-CLAD IL, STA, Milestone Scientific, Inc. Deerfield, IL, USA) and divided by proximal points.

During delivery of the anesthesia, GD raised her hand several times, as a pre-decided sign of pain, and, therefore, the injection was discontinued several times. On the first attempt to place the rubber dam clasp, she complained of pain. Therefore, it was decided to give the supplement anesthesia using a different brand of lidocaine. Thus, she received an addition of $4 / 5$ carpule of octacaine $2 \%$ with 1:100,000 epinephrine, which was also injected intrasulculary, and was effective immediately. After achieving complete anesthesia, GD fully cooperated and treatment was completed uneventfully, to the surprise of her father. On the next appointment (a week later), GD received nitrous oxide analgesia and was anesthetized by $1 \frac{1}{2}$ carpule of octacaine $2 \%$ with 1:100,000 epinephrine, the amount was divided equally between teeth \#8485. The treatment included extraction of 84 and $\mathrm{MO}$ composite restoration on 85 , which was performed uneventfully. During the administration of anesthesia and during treatment, she was fully cooperative.

\section{Case 2}

LY a 13-year-old boy was referred to our clinic because of uncooperative behavior during his previous appointments; he had experienced pain and refused to continue the treatment. After two consecutive, unsuccessful attempts to treat him, he refused to receive dental treatment at all. At the first operative treatment, we attempted to treat teeth \#84 85 (lower right primary molars). Therefore, he was anesthetized by 1 cartridge of lidocaine $2 \%$, 1:100,000 adrenaline in an Intraligamental injection (CCLAD-IL) divided equally between the teeth. Upon placing the clasp of the rubber dam, it was clear that the anesthesia was not effective. Therefore, 1 cartridge of octacaine 2\%, 1:100,000 adrenaline was added and administered by CCLAD-IL; 0.5 cartridge was injected at the buccal aspect and 0.5 to the lingual aspect. Immediately afterward, full anesthesia was achieved, and the treatment was completed uneventfully with full cooperation. Anesthesia of the remaining treated teeth was achieved by a mandibular block to teeth \#36 7475 and infiltration to teeth \#54 55, and by injecting twice the usual volume of local anesthesia and using two brands of local anesthetic together.

\section{Anesthetizing Accessory Innervations, Mixing of Two Brands of Anesthetics (Three Case Reports)}

\section{Case 1}

AD a 12-year-old boy arrived at our clinic because of inability to cooperate, due to his experiencing of pain during previous dental treatments. He arrived at our clinic very frightened but was ready to cooperate. Therefore, we decided to perform his dental treatment under nitrous oxide and to give him beforehand a larger dose of local anesthesia. In an attempt to perform mesio-occlusalbuccal (MOB) restoration in tooth 36 , a mandibular block with 1 cartridge of lidocaine 2\% 1:100,0000 adrenaline, and 1 cartridge of septocaine 3\% 1:200,000 adrenaline was administered by computerized controlled local anesthesia delivery (CCLAD). During the injection, AD fully cooperated but during drilling, he complained of pain. Therefore, an addition of 4/5 cartridge of octacaine was administered Intraligamentary by CCLAD-IL mainly at the distal aspect of the tooth. Immediately afterward, the tooth was effectively anesthetized and the patient fully cooperated. Six months later, following periodic examination, two proximal carious lesions, in upper central incisors, were diagnosed. Taking into consideration his dental history, he was treated under nitrous oxide inhalation. The local anesthetic included 1 cartridge of scandacaine $3 \%$ which was infiltrated into the buccal mucosa and 1 cartridge of octacaine which was divided by two parts, half was injected ligamentary (CCLAD-IL) and half into the incisive canal from the mesial aspect of the palatal papilla. Again, during the injection, AD fully cooperated but during drilling, he complained of pain. Therefore, we added $1 / 2$ cartridge of septocaine 3\% 1:200,000 adrenaline into the incisive canal which was injected this time from the distal aspect of the palatal papilla. Immediately afterward, full anesthesia was achieved and the treatment was completed with full cooperation.

\section{Case 2}

SS a 10-year-old girl was referred to GA because of uncooperation during treatment. Her dental history revealed that 2 months earlier, she had undergone an attempt to treat her upper permanent central incisors. According to her mother, SS cooperated during the delivery of anesthesia but when the drilling started she complained of pain and did not cooperate. This behavior was repeated also when she was treated under conscious sedation. Therefore, she was referred to GA to complete the treatment. Her mother was concerned and looked for a second opinion. On her arrival, SS was introverted and suspicious but cooperated during clinical and radiographic examinations, which revealed two mesial carious lesions in her permanent upper incisors. Taking into account her dental history, it was decided to treat her under conscious sedation. The left central incisor was anesthetized by 1 cartridge of lidocaine 2\%, 1:1,000 epinephrine which was infiltrated to the buccal (3/4 cartridge) and palatal mucosa. During infiltration, SS fully cooperated, but during drilling, at some areas in the cavity, she complained of pain. An addition of Intraligamental anesthesia by lidocaine $2 \%$, 1:1,000 epinephrine did not improve the anesthesia; therefore, the treatment discontinued. At the next appointment, it was decided to inject 1.5 cartridges of lidocaine $2 \%, 1: 1,000$ epinephrine to the buccal mucosa of the teeth and an addition of 0.5 cartridge of lidocaine 2\%, 1:1,000 epinephrine to the incisive canal, half from the mesial aspect of the palatal papilla and half from the distal aspect of the palatal papilla. The anesthesia was effective immediately; SS fully cooperated during the entire treatment.

\section{Case 3}

$\mathrm{OO}$ an 11.5-year-old girl arrived at our clinic fearful and crying. She needed to have tooth 34 extracted for orthodontic purposes. Her mother reported that 3 months earlier, at an attempt to extract the tooth, OO fully cooperated during the administration of the local anesthesia, but during the extraction, she complained 
of pain. She continued to experience pain even after she received supplementary anesthesia at the maximum allowed volume (according to the report of the dentist to her mother). Therefore, the dentist decided to continue with the extraction in spite of her complaints. OO reacted aggressively, ran wild, and screamed. Since then, the girl refused to undergo the extraction of her tooth. On her arrival, she was withdrawn, crying, and avoided eye contact, but gradually she started to interrupt the conversation with some comments and became more relaxed. Therefore, it was decided to extract her tooth at the next appointment, either under nitrous oxide analgesia or under conscious sedation, according to her ability to cooperate. A week later, after she resisted to receive the nitrous oxide inhalation and started to cry, it was decided to perform the extraction under conscious sedation and nitrous oxide inhalation. Immediately before treatment, $\mathrm{OO}$ was more relaxed but completely conscious and still tense and alert. The local anesthesia included 1 cartridge of scandacaine 3\% for inferior alveolar nerve block (including lingual and long buccal) and 1 cartridge of lidocaine $2 \%$ and 1:100,000 adrenaline injected Intraligamentary by CCLAD system. The extraction was performed rapidly and calmly, leaving her astonished as she felt nothing. No additional treatments were needed.

\section{Anesthetizing Accessory Innervation from the Beginning to Prevent Tachyphylaxis, Mixing of Two Brands of Anesthetics (1 Case Report)}

$\mathrm{RO}$, a 12-year-old boy (35 kg) was referred to receive dental treatment under GA because of uncooperative behavior; however, his mother was concerned about the risk of GA and, therefore, asked for a second opinion. His dental history revealed that 2 months earlier, he underwent several attempts to receive dental treatment. First under nitrous oxide inhalation and when these attempts failed, he was referred to receive the treatment under conscious sedation with nitrous oxide-inhalation. According to his mother, RO fully cooperated during the delivery of the local anesthesia; however, he was very sensitive to the vibration of the dental turbine, and, therefore, each time the drilling started, he resisted the treatment. When the treatment attempts continued in spite of his pain, he ran wild.

RO fully cooperated during the clinical and radiographic examinations, which revealed several dentinal caries lesions in teeth \#55, 65, and 75 (primary upper right and left second molar and primary lower left second molar) and completely destroyed tooth \#74 (primary lower left first molar). He was referred to treatments under nitrous oxide analgesia $\left(50 \% \mathrm{~N}_{2} \mathrm{O} / 50 \% \mathrm{O}_{2}\right)$ taking into consideration his previous dental history. At the first operative treatment, we planned to treat the primary upper right second molar \#55. Following nitrous oxide induction, the tooth was anesthetized by local infiltration to the buccal mucosa of the primary second molar and small amount to the buccal mucosa of the first permanent molar to allow placement of a rubber clasp (3/4 carpule of scandacaine $3 \%$ to the buccal mucosa of the primary second molar, $1 / 4$ to the buccal mucosa of the first permanent molar, 2/5 carpule and 1/5 carpule of lidocaine 2\% 1:100,000 adrenaline was injected by CCLAD-IL, to the palatal sulcus of 55 and tooth 16 , respectively). During local anesthesia delivery and during the administration of the rubber dam, the child fully cooperated. However, as the drilling started, the child jumped, raised his hand, and complained of pain. Therefore, 1 cartridge of septocaine $3 \%$, 1:100,000 adrenaline was injected intrasulculary in equal volumes into the buccal and palatal gingiva of the tooth. Similarly, once the drilling started, he jumped and complained again of pain and did not agree to continue. This scenario repeated itself also after performing posterior superior alveolar nerve block by adding 1 cartridge of lidocaine 2\% 1:100,000 adrenaline. Therefore, we decided to discontinue treatment of the child on that day and planned at the next attempt to treat this tooth, to inject the maximum allowed dose of local anesthetic at once, at the beginning of the treatment and to treat him under conscious sedation. At the next appointment ( 2 weeks later), the treatment was performed by conscious sedation ( $10 \mathrm{mg}$ midazolam orally) in combination with nitrous oxide inhalation $\left(50 \% \mathrm{~N}_{2} \mathrm{O} / 50 \% \mathrm{O}_{2}\right)$. In the view that there might be supplemental innervation of tooth \#55 from the previous appointment, it was decided to treat the lower arch (36 sealants, $75 \mathrm{MOD}$ restoration, and extraction of 74). Therefore, the boy received 1 1/4 carpule of lidocaine 2\%, 1:100,000 adrenaline which was injected intrasulculary to teeth \#36, 75, and 74 . Again, during injection and during the administration of the rubber dam, the child fully cooperated, but in the first attempt to drill, he complained of pain. Therefore, we decided to change the type of anesthetic and added 3/5 carpule of septocaine 3\%, 1:100,000 adrenaline through CCLAD-IL. Immediately after completion of anesthesia, the treatment was performed uneventfully, and the child fully cooperated.

On the third appointment ( 3 weeks later), the child was sedated again with $10 \mathrm{mg}$ midazolam orally in combination with nitrous oxide inhalation $\left(50 \% \mathrm{~N}_{2} \mathrm{O} / 50 \% \mathrm{O}_{2}\right)$ for treatment of the primary upper second molar (\#65). With the assumption that there might be a similarity in the innervation of right and left primary second molars (teeth \#55 and 65), and taking into consideration that at the second appointment $O O$ react positively to septocaine, it was decided to inject maximum local anesthetic also to tooth 65 and to inject lidocaine and septocaine. Therefore, the local anesthesia included 1 cartridge of lidocaine 2\%, 1:100,000 adrenaline injected by infiltration to the buccal aspect of the tooth, 1 cartridge of lidocain 2\%, 1:100,000 adrenaline for posterior superior alveolar nerve block and 1 carpule of septocaine $3 \%$ injected into the buccal and palatal gingiva by CCLAD-IL. Again, during the administration of the rubber dam, the child fully cooperated but at the first attempt to drill, he complained of pain. However, after waiting 5 minutes, full anesthesia was achieved, and the treatment was completed uneventfully in a few minutes with full cooperation. On the forth appointment ( 3 weeks later), we attempted again to treat tooth \#55; therefore, following sedation, the child was anesthetized in the same manner of tooth \#65. After waiting 5 minutes, the treatment started and it was completed uneventfully in a few minutes with full cooperation.

\section{Discussion}

Children, who have experienced pain during previous dental treatments, tend to be more fearful and are more likely to demonstrate disruptive behaviors. ${ }^{1-5}$ Fearful children, unlike adults, lack experience acquiring personal resources for managing pain and are, thus, dependent on the skill of their dentist to deal with ineffective anesthesia and to help them enhance their coping skills. ${ }^{3}$

Ineffective anesthesia can be challenging from both a diagnostic and clinical point of view. As fearful children have a lower pain threshold, they tend to complain more about pain during the treatment, and, thus, the reasons for ineffective anesthesia are not always clear. ${ }^{24}$

Therefore, the first imperative approach to prevent paininduced disruptive behavior is to thoroughly assess the reason 
for the disruptive behavior. One of the main observations that can assist the dentist to differentiate between the inability of the child to cooperate and pain related disruptive behaviors is noticing the timing the disruptive behaviors occurs, i.e., full cooperation of the child during the delivery of anesthesia, but the presence of pain-related disruptive behavior during the operative treatment. When disruptive behavior does not occur immediately following the placement of the rubber dam's clasp but only during drilling, it is advisable to exclude other reasons, as anxiety due to noise and vibrations from the dental turbine. This can be done by gradually exposing the child to the increasing intensities of the stimulations. It should be pointed out, however, that treating a child without a rubber dam can increase the negative stimuli during treatment and, thus, increase the probability for disruptive behavior. These triggers include gag reflex (activated by the water, retraction of the tongue or by the cotton roll for isolation of teeth) and toothache from other sensitive nonanesthetized teeth, usually in the opposite arch (for example, teeth with deep caries lesion or $\mathrm{MIH}$ ) that are sensitive to cold water. Obviously, disruptive behavior evoked from these negative stimuli can be prevented by placing a rubber dam.

Ineffective anesthesia can be approached by several means: first, supplement anesthesia to accessory innervation at different locations, depending on the tooth and arch treated. Supplemented anesthesia by Intraligamental anesthesia administered by a computerized delivery system (CCLAD-IL) may be preferred, since it was shown to be as effective as the high-pressure Intraligamental (IL) anesthesia, without its negative consequences of pain during injection, prolonged postoperative pain and development disturbances to the underlying dental buds in young children. ${ }^{17-19,25}$

Second, to inject the maximal allowed dose from the beginning, and to try to anesthetize all the nerve fibers associated with the treated teeth, to prevent tachyphylaxis. Care should be made to not mistakenly correlate between the number of injected cartridges and the achievement of effective anesthesia.

Third, to combine several brands of local anesthetics. It is well known in medicine that in some people, different brands of medicine with the same active ingredient have a different effect. ${ }^{26-30}$ In the present described case series, octacaine $2 \%$ and, in some cases, septocaine $3 \%$ were added following ineffective anesthesia (by lidocaine or tevacaine) and succeeded to induce effective anesthesia. Indeed, it could be argued that any additional dose of local anesthetic would increase the effectiveness of the anesthesia, regardless of the different brand used. However, when the effective dose of one brand does not work, it seems reasonable to inject the additional anesthesia using a different brand, to rule out the possibility that the specific brand is not effective in this specific patient. One subtle suggestion for considering changing the brand of the anesthetic solution was when the child continuously and throughout the injection delivery raised his hand (without disruptive behavior) and complained of pain. Obviously, there is no rule for determining which brand can be more effective, and this requires a trial and error approach.

Another approach is to wait several minutes before initiation of the treatment. Usually, in children, the treatment can be started immediately after injection by CCLAD, due to the spongy bone and the slow delivery of anesthesia. Nonetheless, in one case, we found that waiting 5 minutes following injection improved the effectiveness of the anesthesia.

Obviously, there will still be children that will suffer from ineffective anesthesia even after employing all the aforementioned approaches. These children should be referred to treatment under GA.

\section{Conclusion}

Ineffective anesthesia can lead to severe disruptive behavior when continuing the treatment irrespective to the sensation of pain, as expressed by the child. Several approaches can be used to increase the effectiveness of anesthesia, these include supplementary anesthesia, injecting the maximal dose at once to prevent tachyphylaxis, and changing brands of anesthetics. Adoption of these strategies may decrease the fear associated with dental treatment and reduce the pediatric cases referred to GA.

\section{Clinical Significance}

Behavioral response to ineffective anesthesia may be diagnosed as uncooperative. Effort should be put to differentiate between ineffective anesthesia and uncooperative patient.

\section{References}

1. Allen KD, Kotil D, et al. Comparison of a computerized anesthesia device with a traditional syringe in preschool children. Pediatr Dent 2002 Jul-Aug;24(4):315-320.

2. Gibson RS, Allen K, et al. The Wand vs traditional injection: a comparison of pain related behaviors. Pediatr Dent 2000 NovDec;22(6):458-462.

3. Versloot J, Veerkamp JS, et al. Children's coping with pain during dental care. Community Dent Oral Epidemiol 2004 Dec;32(6):456-461. DOI: 10.1111/j.1600-0528.2004.00187.x.

4. Nakai Y, Milgrom $P$, et al. Effectiveness of local anesthesia in pediatric dental practice. J Am Dent Assoc 2000 Dec;131(12):1699-1705. DOI: 10.14219/jada.archive.2000.0115.

5. Kaufman $E$, Weinstein $P$, et al. Difficulties in achieving local anesthesia. J Am Dent Assoc 1984 Feb;108(2):205-208. DOI: 10.14219/jada. archive.1984.0470.

6. Malamed SF. Neurophysiology. Handbook of local anesthesia, 5th ed., Elsevier Mosby Ino, 2004; p. 25.

7. Childers M, Reader A, et al. Anesthetic efficacy of the periodontal ligament injection after an inferior alveolar nerve block. J Endod 1996 Jun;22(6):317-320. DOI: 10.1016/S0099-2399(96)80267-6.

8. Parirokh M, Satvati SA, et al. Efficacy of combining a buccal infiltration with an inferior alveolar nerve block for mandibular molars with irreversible pulpitis. Oral Surg Oral Med Oral Pathol Oral Radiol Endod 2010 Mar;109(3):468-473. DOI: 10.1016/j.tripleo.2009.11.016.

9. Fan S. Anesthetic efficacy of inferior alveolar nerve block plus buccal infiltration or periodontal ligament injections with articaine in patients with irreversible pulpitis in the mandibular first molar. Oral Surg Oral Med Oral Pathol Oral Radiol Endod 2009 Nov;108(5):e89-e93. DOI: 10.1016/j.tripleo.2009.06.012.

10. Hayden Jr J. The innervation of the maxillary first permanent and primary molars as determined by the deposition of local anesthetic solutions. A preliminary report. Acta Odontol Scand 1965 Apr;23(2):147-162. DOI: 10.3109/00016356509033571.

11. Meyer TN, Lemos LL, et al. Effectiveness of nasopalatine nerve block for anesthesia of maxillary central incisors after failure of the anterior superior alveolar nerve block technique. Braz Dent J 2007;18(1):69-73. DOI: 10.1590/S0103-64402007000100015.

12. Malamed ST. Technique for mandibular anesthesia. Handbook of local anesthesia, 5th ed, Elsevier Mosby, 2004; p. 227.

13. Walton RE, Abbott BJ. Periodontal ligament injection: a clinical evaluation. J Am Dent Assoc 1981 Oct;103(4):571-575. DOI: 10.14219/ jada.archive.1981.0307.

14. Smith GN, Walton RE, et al. Clinical evaluation of periodontal ligament anesthesia using a pressure syringe. J Am Dent Assoc 1983 Dec;107(6):953-956. DOI: 10.14219/jada.archive.1983.0357.

15. Cohen HP, Cha BY, et al. Endodontic anesthesia in mandibular molars: a clinical study. J Endod 1993 July;19(7):370-373. DOI: 10.1016/S00992399(06)81366-X. 
16. Nusstein J, Claffey E, et al. Anesthetic effectiveness of the supplemental intraligamentary injection, administered with a computer-controlled local anesthetic delivery system, in patients with irreversible pulpitis. J Endod 2005 May;31(5):354-358. DOI: 10.1097/01.DON.0000140565.88940.60.

17. Brannstrom M, Lindskog S, et al. Enamel hypoplasia in permanent teeth induced by periodontal ligament anesthesia of primary teeth. J Am Dent Assoc 1984 Nov;109:735-736. DOI: 10.14219/jada.archive.1984. 0172.

18. Ashkenazi M, Blumer $\mathrm{S}$, et al. Effect of computerized delivery intraligamental injection in primary molars on their corresponding permanent tooth buds. Inter J Paediatr Dent 2010 July;20:270-275. DOI: 10.1111/j.1365-263X.2010.01049.x.

19. Ashkenazi M, Blumer S, et al. Post-operative pain and use of analgesic agents in children following intrasulcular anesthesia and various operative procedures. Br Dent J 2007 Mar;202:E13. DOI: 10.1038/ bdj.2007.81.

20. Wright GZ, Starkey PE, et al. Local anesthesia in children. Managing children's behavior in the dental offices; 1983. pp. 126-139.

21. Fukayama H, Yoshikawa F, et al. Efficacy of anterior and middle superior alveolar (AMSA) anesthesia using a new injection system: the Wand. Quintessence Int 2003 July-Aug;34(7):537-541.

22. Rodd HD, Boissonade FM, et al. Pulpal status of hypomineralized permanent molars. Pediatr Dent 2007 Nov-Dec;29(6):514-520.

23. Fagrell TG, Lingström $P$, et al. Bacterial invasion of dentinal tubules beneath apparently intact but hypomineralized enamel in molar teeth with molar incisor hypomineralization. Int J Paediatr Dent 2008 Sep;18(5):333-340. DOI: 10.1111/j.1365-263X.2007.00908.x.

24. Versloot J, Veerkamp JS, et al. Pain behaviour and distress in children during two sequential dental visits: comparing a computerised anaesthesia delivery system and a traditional syringe. Br Dent J 2008 July 12;205(1):E2. DOI: 10.1038/sj.bdj.2008.414.

25. Ashkenazi M, Blumer $\mathrm{S}$, et al. Effectiveness of various modes of computerized delivery of local anesthesia in primary maxillary molars. Pediatr Dent 2006 Jan-Feb;28(1):29-38.

26. Bele MH, Derle DV. Effect of sorbed water on disintegrant performance of four brands of Polacrilin Potassium NF. APS Pharm Sci Tech 2012 Mar;13(1):24-34. DOI: 10.1208/s12249-011-9717-8.

27. Feldmeyer $L$, Benden $C$, et al. Not all intravenous immunoglobulin preparations are equally well tolerated. Acta Derm Venereol 2010 Sep;90(5):494-497. DOI: 10.2340/00015555-0900.

28. Mittal G, Kumar N, et al. A radiometric study of factors affecting drug output of jet nebulizers. Indian J Pharm Sci 2010 Jan;72(1):31-38. DOI: 10.4103/0250-474X.62234.

29. Lamprecht $\mathrm{G}$. In vitro determination of the release of alendronic acid from alendronate tablets of different brands during deglutition. J Pharm Sci 2009 Oct;98(10):3575-3581. DOI: 10.1002/jps.21639.

30. Datta P, Dasgupta A. Effect of Chinese medicines Chan Su and Danshen on EMIT 2000 and Randox digoxin immunoassays: wide variation in digoxin-like immunoreactivity and magnitude of interference in digoxin measurement by different brands of the same product. Ther Drug Monit 2002 Oct;24(5):637-644. DOI: 10.1097/00007691-200210000-00010. 